
\title{
\& Research Square \\ Life goes on: How cosmic doomsday might not be the end
}

David Sloan

Rafael Alves Batista

Abraham Loe

\section{Video Abstract}

Keywords: Scientific Reports, University of Oxford, resilience, life, astrophysical events, Earth-like planet, gamma-ray burst, asteroid, supernova, passing-by star, extremophile, tardigrade, impact winter, mass extinction, radiation, cumulative impact rate, planetary system, Solar system, exoplanetary life, sterilization

Posted Date: September 20th, 2019

DOI: https://doi.org/10.21203/rs.2.15061/v1

License: (c) (i) This work is licensed under a Creative Commons Attribution 4.0 International License. Read Full License 


\section{Abstract}

A team of researchers from the US and the UK has launched a mathematical assault on our planet. Using energy calculations, they've tasked themselves with determining the ability for life as we know it to make it through a catastrophe of cosmic proportions. It's the end of the world as we know it. And the verdict is that life will be fine. Just what kind of calamity would it take to wipe out all life? The simplest answer is that, since life as we know it depends on water, the entire ocean would have to boil. Earth's toughest creature, the tardigrade, can withstand temperatures well above boiling. But it can do so for only a few minutes. So the question now becomes, what cosmic events are cataclysmic enough to vaporize our oceans? Mathematically, it's an energy problem-to which there are three possible answers. The first is an asteroid impact. Many space objects have struck Earth throughout history. And many still might. Even so, only 17 known asteroids within our solar system are massive enough to cause the oceans to evaporate. And the probability of that happening is incredibly tiny. Based on the rate at which asteroids impact Earth and extrapolating to doomsday-level asteroid sizes, a life-obliterating object is likely to make contact only once every 100 million billion years-well beyond the age of the universe. The next possible scenario is a supernova. A bursting star sends out an enormous shockwave carrying more than enough energy to destroy all life on our planet. But to absorb that energy, Earth would have to be sufficiently close to the blast, or around one-hundredth of a light year away. How likely is that? Consider that Earth's nearest star, besides the sun, is Alpha Centauri, which lies just over 4 light years away and is not the right type of star to go supernova. Finally, there's the possibility of a fatal gamma ray burst. These violent explosions are considered the strongest electromagnetic events throughout the known universe. As such, Earth could be as far as 42 light years away from a gamma ray jet and still see all life perish. Fortunately, the probability of such an event affecting our planet or others like it is extremely small, at about once every 10 billion years. It should be noted that these calculations assume only ocean boiling as the end result. It is entirely possible that a less energetic event could trigger a secondary catastrophe that spells the end of life as we know it. Still, the exercise should serve as a source of hope-not only for the continued survival of life here on Earth but also for possible life beyond. 